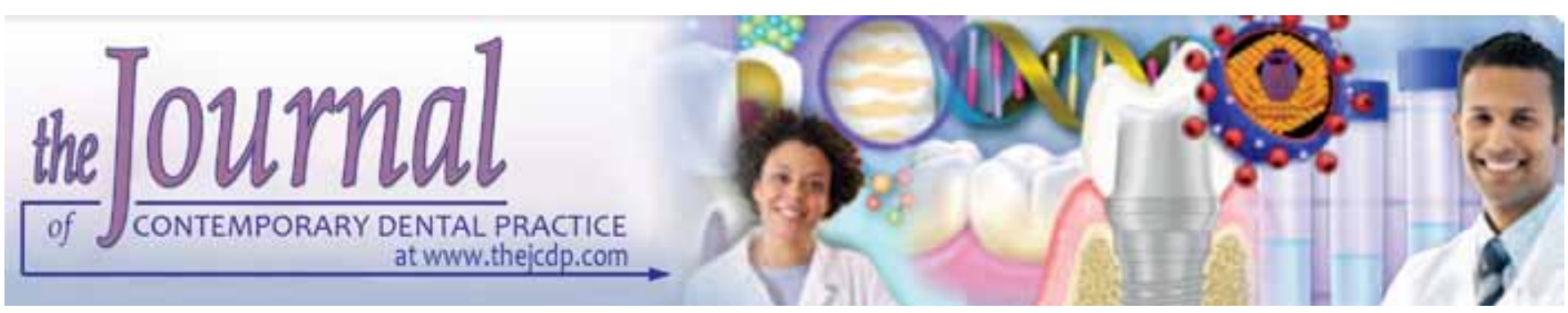

\title{
Smokeless Tobacco-associated Lesions: A Mobile Health Approach
}

\author{
${ }^{1}$ Rani Desai, ${ }^{2}$ Praveen Birur, ${ }^{3}$ Suchitra Bajaj, ${ }^{4} \mathrm{AR}$ Shubhasini, ${ }^{5} \mathrm{R}$ Bhanushree, ${ }^{6} \mathrm{G}$ Shubha, ${ }^{7} \mathrm{G}$ Keerthi, ${ }^{8}$ Vidya Tiwari \\ ${ }^{9}$ Sanjana Patrick, ${ }^{10}$ Usha Hegde, ${ }^{11}$ Sujit Shetty, ${ }^{12}$ Vidya Nagabhushan, ${ }^{13}$ Sandeep Kankanala, ${ }^{14}$ Shreyas Shah
}

\begin{abstract}
Aim: Globally, India accounts for the highest number of oral cancer cases. The survival rates are about $30 \%$ lower than those in developing countries. The main reason for these dismal figures is the late presentation of patients. In order to downstage oral cancer in such a scenario, screening and diagnosis at an early stage is warranted. A pragmatic approach is needed for an oral cancer screening program, hence a mobile health (mHealth) approach was used. In this approach, health workers were empowered with mobile phones with decision-based algorithm. Risk stratification of tobacco habit enables us to identify lesions associated with particular habits.
\end{abstract}

Materials and methods: A specific cohort of factory employees who predominantly had pure tobacco chewing habit was chosen to examine the effect of pure tobacco on oral mucosa. One thousand three hundred and fifty-seven subjects were screened in two phases. In the first phase, habits and oral lesions were identified and photographed. The photographs were remotely diagnosed by an oral medicine specialist and those subjects requiring biopsy were recalled for phase II. Cytology and biopsy were performed in phase II.

\footnotetext{
1,3,8,9,12Biocon Foundation, Bengaluru, Karnataka, India

${ }^{2}$ Department of Oral Medicine and Radiology, Biocon Foundation KLE Society's Institute of Dental Sciences, Bengaluru Karnataka, India

4-7,13 Department of Oral Medicine and Radiology, KLE Society's Institute of Dental Sciences, Bengaluru, Karnataka, India

${ }^{10}$ Department of Oral and Maxillofacial Pathology, JSS Dental College and Hospital, Mysuru, Karnataka, India

${ }^{11}$ Department of Oral and Maxillofacial Surgery, JSS Dental College and Hospital, Mysuru, Karnataka, India

${ }^{14}$ Department of Oral Medicine and Radiology, Bharati Vidyapeeth Dental College, Navi Mumbai, Maharashtra, India

Corresponding Author: Praveen Birur, Professor and Head Department of Oral Medicine and Radiology, Biocon Foundation KLE Society's Institute of Dental Sciences, Bengaluru Karnataka, India, e-mail: praveen_birur@yahoo.com
}

Results: The predominant habit was smokeless tobacco (SLT), in 582 subjects. The most commonly encountered lesion was tobacco pouch keratosis seen in 397 subjects. Biopsy was performed for 71 subjects, most cases showed hyperkeratosis and mild dysplasia. One subject had moderate dysplasia.

Conclusion: There was minimal alteration of tissues in our study subjects, which can be considered as low-risk. Use of mHealth empowered frontline healthcare workers to identify subjects with lesions and enabled remote diagnosis by specialist in resource-constrained settings.

Clinical significance: Use of mHealth enabled us have an electronic record of subject details. This data shall be used for a planned follow-up of the same cohort after 3 years.

Keywords: Cohort study, Mobile-health, Smokeless tobacco, Tobacco pouch keratosis.

How to cite this article: Desai R, Birur P, Bajaj S, Shubhasini AR, Bhanushree R, Shubha G, Keerthi G, Tiwari V, Patrick S, Hegde U, Shetty S, Nagabhushan V, Kankanala S, Shah S. Smokeless Tobacco-associated Lesions: A Mobile Health Approach. J Contemp Dent Pract 2015;16(10):813-818.

Source of support: Nil

Conflict of interest: None

\section{INTRODUCTION}

Oral cancer is a leading cause of morbidity and mortality. Globally, the annual incidence is approximately 275,000 . Retrospective analysis has shown increase in the rate of occurrence every year. ${ }^{1}$ Oral cancers are usually preceded by clinically evident oral potentially malignant disorders (OPMDs). ${ }^{2}$ Thus, it is important to monitor and evaluate the progression of OPMDs to downstage cancer.

Most oral cancers are largely preventable, if detected early. Screening through direct visual examination of the oral cavity is a cost-effective method and an important intervention in oral cancer control. ${ }^{3}$ However, in resource-constrained settings, an mobile health (mHealth) (mobile-based oral screening) module with decision supported algorithm was conceived for early detection 
of oral lesions by frontline healthcare workers. With this objective, mobile early detection and prevention of oral cancer program was carried out to reach out to maximum number of individuals.

Tobacco and areca nut are the most important risk factors for oral cancer. Tobacco is available as smoked and smokeless tobacco (SLT). The types of SLT placed in oral cavity are loose-leaf chewing tobacco, moist snuff, and dry snuff. ${ }^{4}$ In India, SLT usage is a sociocultural milieu in various communities. There is regional variation in the availability of SLT, ranging from unflavored tobacco, traditional betel quid, to flavored forms, such as Khaini, Zarda, Mishri, Datun, etc. Areca nut is the main ingredient in Gutkha and Mawa, with traces of tobacco and other chemicals and flavoring agents. ${ }^{5}$

Smokeless tobacco, as opposed to smoked tobacco, has direct contact with oral mucosa. The product type, site of placement, frequency and duration of exposure are important factors which cause mucosal alterations. ${ }^{5}$ Oral mucosal lesions observed in association with SLT are smokeless tobacco pouch keratosis and other OPMDs like leukoplakia and erythroplakia. ${ }^{6}$

The early detection and prevention of oral cancer program was conducted to evaluate the effect of tobacco on oral mucosa and to correlate with the histopathology among tobacco habituated population.

\section{MATERIALS AND METHODS}

Institutional ethics committee provided approval for the project. Written informed consent was obtained from all the participants.

The study sample: The early detection and prevention of oral cancer screening program was divided into two phases. Phase I was oral examination to identify the tobacco related oral lesions. Phase I participants were all employees enrolled in the registry of six different factories in one town.

Phase I: The target population was in a workplace setting. This population predominantly had a pure, nonflavored tobacco chewing habit. The participants' age was 21 years or over. Recruitment for the oral examination screening was done in batches; screening was done in their work place and during their working hours with minimal disturbance of their production work. The phase I screening was done for 9 days.

Four health workers and a dentist were involved in Phase I screening. All the health workers and the dentist were trained to identify the oral lesions and all had an experience of 3 years in oral cancer screening.

mHealth: Subjects were identified based on their provident fund number as registered in the company records. Phase I screening was aided with a mobile phone that had a decision-based algorithm, point of interest (POI) Mapper algorithm. Demographic details and status of health insurance were obtained. Emphasis was given to identify type of habits, such as flavored or nonflavored tobacco, gutkha, areca nut, alcohol and combination of products along with frequency and duration of use, using specific questions.

All subjects underwent a comprehensive examination of oral mucosa. The examination was undertaken using a chair with proper head and backrest under a good natural light and a torch as an additional light source. Disposable mouth mirrors and probes were used. With the aid of mobile phone, subjects were interviewed and stratified to high-or low-risk group. Subjects giving an affirmative response to any of the following questions were considered to be high-risk; age above 40, tobacco use, history of long standing ulcer or swelling in head and neck region, and family history of cancer.

The high-risk individuals underwent mapping of the oral cavity in a predefined fashion and photographs of oral lesions were obtained using the mobile phone. All subjects who were positive for tobacco usage were given anti-tobacco counseling and put on rigid follow-up. Lowrisk individuals were encouraged to participate in tobacco awareness program and then discharged.

All the data obtained through the mobile phones was uploaded to an open-source medical record system (open MRS). An oral medicine specialist of the central coordinating center then reviewed the data and photographs of the lesions. A remote diagnosis was provided for each subject, as homogenous or nonhomogenous leukoplakia, erythroplakia, tobacco pouch keratosis, smokers melanosis, OSF, or carcinoma. Based on this, biopsy was recommended when required.

Notifications with follow-up instructions were sent to the respective health workers. On receiving the message the health worker identified the patients for phase II. Participants who were positive for tobacco usage but had not developed oral lesions were also included in the phase II for on-site re-evaluation by specialist.

Phase II: This phase consisted of on-site evaluation by an oral medicine specialist and biopsy. A second written informed consent was obtained for phase II. All subjects who had a tobacco habit were recalled for phase II of the study. Smears were obtained from the surface of the lesion for exfoliative cytology and liquid based cytology (LBC). Punch biopsy using $5 \mathrm{~mm}$ disposable punch was subsequently performed. The smears and biopsy were performed by a team of eight dentists under the guidance of an oral and maxillofacial surgeon. 


\section{RESULTS}

Total combined employees in the six factories were 2400. One thousand three hundred and fifty-seven employees were available for participation in the screening program. Out of the 1,357 subjects, 1083 were males (Table 1). Based on Kuppuswamy criteria (2014), ${ }^{7} 379$ belonged to lower socioeconomic strata (Table 2). The age group was between 21 and 59 years with majority in third and fourth decade.

Data collection in terms of habit details was invalid in 83 subjects and was not incuded in analysis. Eight hundred sixty-four subjects gave a current or past habit history (Table 3). Subjects using flavored tobacco, nonflavored tobacco, and mishri were grouped as having SLT habit. Subjects using areca nut, pan masala and gutkha were grouped as areca nut chewers. Subjects with history of chewing any of tobacco products and areca nut containing products were grouped as having a combination of habits.

The most prevalent habit was SLT, in 582 subjects. Forty-two subjects used areca nut products. Two hundred twenty-six subjects used a combination of tobacco and areca nut products. Fourteen subjects smoked tobacco or consumed alcohol alone without the habit of SLT use.

Out of these 864 subjects, 582 were detected as having oral lesions. Two hundred and eighty-two subjects had no lesions (Table 4).

Table 1: Gender distribution

\begin{tabular}{lll}
\hline Gender & $n$ & Percentage \\
\hline Male & 1083 & 80 \\
Female & 274 & 20 \\
\hline Total & 1357 & 100 \\
\hline
\end{tabular}

Table 2: Socioeconomic status

\begin{tabular}{lll}
\hline SE status & $n$ & Percentage \\
\hline$\leq 1802$ & 1 & 0.10 \\
$1803-5386$ & 75 & 7.18 \\
$5387-8988$ & 379 & 36.27 \\
$8989-13894$ & 285 & 27.27 \\
$13495-17999$ & 113 & 10.81 \\
$18000-36016$ & 178 & 17.03 \\
$\geq 36017$ & 14 & 1.34 \\
\hline Total & 1045 & 100 \\
\hline
\end{tabular}

The 582 oral lesions were photographed and uploaded. Remote diagnosis was peformed by an oral medicine specialist. Out of the 582 uploads, 43 did not have photographs of lesions, and eight had poor quality photographs. However, risking overdiagnosis, all subjects with habits were recalled for phase II of the study.

The most common diagnoses were tobacco pouch keratosis and homogenous leukoplakia, 397 and 79 in number respectively. The comparison of the habit type and associated lesions are shown in (Table 5). The mean frequency and mean duration were higher among subjects with lesions when compared to those without (Tables 4 and 5).

Four hundred and sixty-one of subjects had lesions in the buccal mucosa with predilection for leftside in 346 cases.

Phase II of the study was conducted after 3 weeks. All subjects with a habit history were recalled for onsite screening by oral medicine specialist, however, only 317 of them presented for examination. The initial diagnosis was reviewed for these subjects. Twelve subjects, who had been diagnosed with tobacco-related lesions were found to have no oral lesions, and 12 more were found to have OSF.

Of all subjects screened, 71 consented for biopsy. Smears were obtained and biopsy was performed at the site of the lesion (Table 6).

Histopathology revealed the most common diagnosis to be hyperkeratosis in 47 subjects followed by mild

Table 3: Total number of subjects with habits

\begin{tabular}{ll}
\hline Habit & Number \\
\hline Smokeless tobacco & 582 \\
Areca nut & 42 \\
Combination & 226 \\
Other & 14 \\
\hline Total & 864 \\
\hline
\end{tabular}

Table 4: Details of subjects with habits without lesions

\begin{tabular}{llll}
\hline Habit & Number & $\begin{array}{l}\text { Mean frequency } \\
\text { (times/day) }\end{array}$ & $\begin{array}{l}\text { Mean duration } \\
\text { (years) }\end{array}$ \\
\hline Smokeless tobacco & 187 & 3.8 & 9.3 \\
Areca nut & 29 & 2.7 & 3.1 \\
Combination & 56 & - & - \\
Other & 10 & - & - \\
\hline Total & 282 & & \\
\hline
\end{tabular}

Table 5: Details of subjects with lesions

\begin{tabular}{|c|c|c|c|c|c|c|c|c|}
\hline Habit & Number & $\begin{array}{l}\text { Mean frequency } \\
\text { (times/day) }\end{array}$ & $\begin{array}{l}\text { Mean duration } \\
\text { (years) }\end{array}$ & $\begin{array}{l}\text { Tobacco pouch } \\
\text { keratosis }\end{array}$ & Leukoplakia & OSF & $\begin{array}{l}\text { Other } \\
\text { lesion }\end{array}$ & $\begin{array}{l}\text { Multiple } \\
\text { lesions }\end{array}$ \\
\hline Smokeless tobacco & 395 & 4.2 & 12.1 & 293 & 53 & 0 & 24 & 25 \\
\hline Areca nut & 13 & 2.5 & 5.3 & 0 & 1 & 10 & 0 & 2 \\
\hline Combination & 170 & - & - & 100 & 25 & 13 & 12 & 20 \\
\hline Other habit & 4 & 一 & - & 4 & - & - & - & - \\
\hline Total & 582 & & & 397 & 79 & 23 & 36 & 47 \\
\hline
\end{tabular}


Table 6: Biopsy details

\begin{tabular}{llllll}
\hline Lesion & Number & Hyperkeratosis & Mild dysplasia & Moderate dysplasia & Inconclusive \\
\hline Tobacco pouch keratosis & 56 & 35 & 13 & 1 & 7 \\
Homogenous leukoplakia & 10 & 8 & 2 & - & - \\
Non homogenous leukoplakia & 3 & 2 & - & - & 1 \\
Erythroplakia & 2 & 2 & - & - & - \\
\hline Total & 71 & 47 & 15 & 1 & 8 \\
\hline
\end{tabular}

dysplasia in 15 subjects and moderate dysplsia in one subject. Eight cases were inconclusive. One case had no tissue available for histopathological analysis, others showed only salivary acini, muscle tissue, or Fordyce's granules.

\section{DISCUSSION}

Rising longevity, alterations in life styles and progressive control of communicable diseases has led to emergence of cancer and noncommunicable diseases as an important health problem in India and other developing countries. Statistics from ICMR state that the burden of cancer cases is expected to double by the year $2016 .^{8}$ The age standardized cancer mortality rate per $1,00,000$ population for lip, oral cavity and pharynx was 22.1 as reported in the million death study. ${ }^{9}$

The overall survival of Indian patients with cancer is about $30 \%$ lower than the developed countries and its economic fallout is devastating. Data from regional cancer centers in India suggest that lower survival is primarily due to delayed diagnosis and presentation in late stages. More than two-thirds of patients report in an advanced stage at the time of diagnosis. Lack of awareness, inadequate diagnostic services, and lack of trained specialists leads to delayed diagnosis. Presentation in advanced stage results in low treatment outcomes and higher cost to patients.

There is evidence that direct visual examination of the oral cavity is a cost-effective method for oral cancer screening in resource-constrained settings. ${ }^{3}$ However, conventional screening has a few limitations. Poor patient compliance to follow-up after screening is one of the main drawbacks. The other limitations include lack of permanent portable record of data after screening, slow rates of information transfer, poor communication between primary provider and specialist. Rigid follow-up to monitor the progression of OPMD is very critical in oral cancer surveillance program.

mHealth in oral cancer screening: To address the shortcomings in conventional screening, we introduced an mHealth program. Biocon Foundation, a voluntary nongovenrnment organization, has developed a mobile phone-based management platform for cancer screening and surveillance. This platform enables the creation of electronic health records and facilitates targeted screening for oral cancer. The implementation of this project design aims at empowering frontline healthcare workers with mobile phone equipped with decision-based algorithm for early detection of oral cancer. This mHealth program provides a platform to connect specialists with rural population for diagnosis and also creates an opportunity for follow-up and referrals.

Another important feature of the program is electronic data capture (EDC). It helps in capturing necessary information and pictures of oral cavity. This data are uploaded in the openMRS. Open-source medical record system, is a platform for data storage accessed by specialist to assess the lesions even from a remote location. The screening process is evidence based, as all data have individual identifying numbers correlated with their tobacco history and clinical photographs. The procedure is low cost and it replaces visual inspection by a specialist enabling community health worker to conduct the screening procedure. The project aims at reducing oral cancer burden and improve the treatment outcome and financial liabilities. It believes that use of technology for oral cancer screening would decrease the cost burden on the healthcare machinery.

The final goal of the early detection and prevention of oral cancer program is to monitor the progression of the OPMD. We have developed a good surveillance program by using mHealth and local health workers. Intraoral photographs, which are captured during data collection, are used for education and awareness. We have found this very effective way to teach self-mouth examination. This has provided a personalized approach to high-risk individuals. Electronic data also aid in follow-up of cases.

The biggest challenge in oral cancer control is to deaddict the tobacco usage. Most of our rural population is heavily dependent on tobacco both in chewing and smoking forms. The low economic status and the monotonous work have resulted in heavy dependency of tobacco among these people. Most of these individuals use tobacco products to break the monotony during their routine work, rather than for pleasure. Hence habit cessation efforts in most of these individuals fail, posing a big challenge in cancer control program.

Smokeless tobacco and oral lesions: Oral cancer is primarily caused by the use of tobacco and its products, which are common practices in rural India. Tobacco related cancers constitute $42 \%$ of all cancers in males. ${ }^{9}$ The present study 
population predominantly chewed tobacco without any flavoring agent. We assessed any mucosal alteration among these subjects.

Eighty percent of the subjects were males. This high preponderance was because the study was conducted in a workplace setting dominated by a male work force. In most countries around the world, oral cancer is more common in men than women. The reported gender differences are attributable to heavier indulgence in risk habits (tobacco and alcohol) by men and exposure to sunlight (for lip cancer) as a part of outdoor occupations. ${ }^{10}$ Most of our subjects were in the third and fourth decade of life.

Around $63.6 \%$ of subjects in our study had an adverse habit. Among Indian adults, the prevalence of tobacco use is about $35 \%$. Our study subjects chewed tobacco which was available as flakes in pouches. Some of them may have combined it later with slaked lime.

Most of our subjects chewing nonflavored tobacco had a habit duration of 3 to 5 years and chewed 5 to 10 times a day. As duration of tobacco habit increases, there are more chances of development of precancerous lesions. ${ }^{4}$ Oral mucosa may exhibit various changes on long-term contact of tobacco which could be due to the carcinogen itself or as a protective mechanism of the oral cavity. These changes can be categorized as tobacco-induced oral mucosal lesions which are less likely to cause cancer, lesions that are potentially malignant. ${ }^{11}$

No lesions were found among subjects who had no habit. Around $68.2 \%$ of all lesions detected were tobacco pouch keratoses, and $13.5 \%$ were homogenous leukoplakias. A few lesions of tobacco pouch keratosis were scrapable, this could have been associated with excessive use of slaked lime causing erosions of the lesion. Eight percent of subjects had multiple lesions, of which tobacco pouch keratosis was most commonly encountered.

The high level of oral mucosal soft tissue lesions may be attributed to the irritational effect of tobacco on oral structures, including genetic and host modification factors.

Similar results were reported by Shanker et al, ${ }^{12}$ Ambedkar et al, ${ }^{5}$ Al-Attas et al, ${ }^{13}$ Lesan et $\mathrm{l}^{14}$ and Chitroda et $\mathrm{al}^{15}$ who found a high prevalence of mucosal lesions in subjects with SLT habits. Al-Mufti et al conducted a narrative systematic review to determine the type of oral mucosal lesion caused by different types of SLT and concluded that some types of SLT products tend to be associated with specific types of oral lesions at the site where the product is placed. ${ }^{16}$

Chitroda et al found that SLT induced lesions were more common than leukoplakia in their subjects and reasoned that SLT induced lesions develop sooner due to local irritation caused by the placement of quid. They also found that SLT induced lesions were more in number when tobacco was used wih lime. ${ }^{15}$

Smokeless tobacco keratosis becomes apparent after 5 to 10 years of tobacco habit initiaion and regresses with cessation of habit. The development of tobacco pouch keratosis is dependent on the type of habit popular in a society. Keratosis occurs more frequently in snuff users rather than tobacco chewers, and when quid is placed at one site rather than multiple sites. The specific brand of tobacco used, extended duration of habit, increased frequency of use, excessive daily contact-hours of tobacco on oral mucous membranes, and a deficiency of betacarotene or vitamin A also contribute to a high risk of keratosis. $^{17}$

Smears obtained in the second phase of our study were analyzed. Both Exfoliative cytology and LBC did not show any atypical cells. But, the LBC technique showed better cytomorphological features without overlapping of cells and a clear background as against conventional exfoliative cytology. Liquid based cytology as 'point of care diagnosis' needs to be assessed as it is noninvasive. These findings are similar to other studies. ${ }^{18,19}$

Punch biopsy was performed in 71 subjects. Around $66 \%$ of them had hyperkeratosis, $19.7 \%$ had mild and only $1.4 \%$ had moderate dysplasia. All subjects with dysplasia are under rigid follow-up.

Malignant transformation of oral leukoplakias has been reported in the range of 1 to $20 \%$ over 1 to 30 years. There is a distinct difference between tobacco pouch keratosis and oral leukoplakia with respect to the presence of epithelial dysplasia, which is more frequently found in the latter. The degree of dysplasia is also of a milder nature in tobacco pouch keratosis. ${ }^{6}$

The carcinogenic potential of SLT has been a subject of cosiderable debate, and no global consensus has been reached. The relative risk of developing oral and upper respiratory cancers with use of moist snuff and chewing tobacco ranges from 0.6 to 1.7; while it ranges from 4 to 13 with dry snuff use. The risks from SLT, unspecified as to type, range from 1.5 to $2.8 .^{20}$

Subjects with lesions had a higher frequency and greater duration of habit than those subjects without lesions. Aruna et al suggested that duration of risk habit was more important than frequency in determining the risk for oral cancer causation. However, both the frequency of the habit and duration are important in determining the severity of effects in relation to oral cancer. ${ }^{21}$

Gupta et al conducted a systematic review and noted a linear dose-response relationship between the number of tobacco quids chewed per day and the risk of oral cancer. $^{22}$

Some limitations of our study include invalid data collection of habit use. This data were collected by health 
workers, in a community-based setting, and in view of the large population assessed, some errors are anticipated. The invalid data was not included in statistical analysis. Subjects with no history of habit were not re-evaluated by the oral medicine specialist. It is possible a few of them may have had mucosal alterations. Further, there may have been under-reporting of habit history by participants. There were some drawbacks in tissue biopsy, with eight cases being inconclusive.

Our research is focused on risk stratification of different tobacco products, areca nut and their combinations. We have phased our data collection from cohorts depending on tobacco products. Further studies are required to address the presence and severity of OPMDs in subjects with other forms of tobacco and areca nut chewing habits.

This study had a specific sample collection from tobacco chewers. The predominant age group on this study was between 30 and 40 years of age. Though the duration of chewing ranged from 2 to 30 years, the mucosal alteration was minimal. These results indicate a very minimal alteration of tissues, which cannot to be categorized under OPMDs and can be considered as low-risk. Similar results have been reported by Al-Mufti et al, ${ }^{16}$ Al-Attas et $\mathrm{al}_{1}{ }^{13}$ Chitroda et al. ${ }^{15}$ We intend to follow-up these subjects after a period of 3 years to examine the effectiveness of tobacco cessation counseling and assess any progress in lesions. It is critical to detect these lesions early and have a rigid follow-up of OPMDs.

Clinical significance: This mHealth based study was conducted in a workplace setting among subjects who predominantly chewed pure tobacco. Clinical examination and biopsy for selected subjects was performed. Minimal mucosal alteration was observed. mHealth approach enabled us to maintain electronic data and follow-up the subjects.

\section{REFERENCES}

1. Warnakulasuriya S. Living with oral cancer: epidemiology with particular reference to prevalence and life-style changes that influence survival. Oral Oncol 2010;46:407-410.

2. Ho PS, Chen PL, Warnakulasuriya S, Shieh TY, Chen YK, Huang Y. Malignant transformation of oral potentially malignant disorders in males: a retrospective cohort study. BMC Cancer 2009;9:260.

3. Sankarnarayanan R, Ramadas K, Thomas G, Muwange R, Thara S, Mathew B, et al. Effect of screening on oral cancer mortality in Kerala, India: a cluster-randomized controlled trial. Lancet 2005;365:1927-1933.

4. Rodu B, Jansson C. Smokeless tobacco and oral cancer: a review of the risks and determinants. Crit Rev Oral Biol Med 2004;15:252-263.

5. Ambedkar DM, Chaudhary BJ, Kulkarni VV. A study of oral precancerous lesions in relation to tobacco habituation. Int J Med Clin Res 2014;5:282-285.
6. Greenberg MS, Glick M, Ship JA. Burket's Oral Medicine. 11th ed. BC Decker Inc 2008. p.100-101.

7. Maheshwaran G. Kuppuswamy's socioeconomic status scale: a revision of income parameter for 2014. Int J Recent Trends Sci Technol 2014;11:1-2.

8. Murthy NS, Chaudhry K, Rath GK. Burden of cancer and projections for 2016, Indian scenario: gaps in the availability of radiotherapy treatment facilities. Asian Pac J Cancer Prev 2008;9:671-677.

9. Dikshit R, Gupta PC, Ramasundarahettige C, Gajalakshmi V, Aleksndrowicz L, Badwe R, et al. Cancer mortality in India: a nationally representative survey. Lancet 2012;379:2343-2351.

10. Warnakulasuriya S. Living with oral cancer: epidemiology with particular reference to prevalence and lifestyle changes that influence survival. Oral Oncol 2010;46:407-410.

11. Sridharan G. Epidemiology, control and prevention of tobacco induced oral mucosal lesions in India. Ind J Cancer 2014;51: 80-85.

12. Shankar A, McMunn A, Steptoe A. Health-related behaviors in older adults in relationship with socioeconomic status. Am J Prev Med 2010;38:39-46.

13. Al-Attas SA, Ibrahim SS, Amer HA, Darwish ZE, Hassan MH. Prevalence of potentially malignant oral mucosal lesions among tobacco users in Jeddah, Saudi Arabia. Asian Pac J Cancer Prev 2014;15:757-762.

14. Lesan S, Nosratzehi T, Ousia MA, Arbabikalati F, Pourmardan E. The correlation between the frequency of oral lesions and the amount of smokeless tobacco usage in patients referred to oral medicine department Zahedan dental school. J Dent Shiraz Univ Med Sci 2014;15:81-85.

15. Chitroda PK, Shah JT, Katti G, Ghali S. A correlative study of smokeless tobacco-induced lesion and smoke-induced leukoplakia in various aspects. J Ind Acad Oral Med Radiol 2011;23:86-91.

16. Al-Mufti H, Batley H, Ali M, Heshmat H, Ahmed F. Do different types of smokeless tobacco products cause differing or similar oral lesions? Face Mouth and Jaw Surgery. Int Trainee J Oral Maxillofac Surg 2014;4:1-9.

17. Bouquot JE, Speight PM, Farthing PM. Epithelial dysplasia of the oral mucosa-Diagnostic problems and prognostic features. Current Diag Pathol 2006;12:11-21.

18. Campagnoli ED, Sandrin R, França BHS, Sassi LM, Machedo MAN, Lima AAS. Comparison between liquid-based cytology and conventional exfoliative cytology in the diagnosis of head and neck carcinomas. Braz Res Pediatr Dent Integr Clinic 2011;11(1):65-71.

19. Dwivedi N, Agarwal A, Raj V, Kashyap B, Chandra S. Comparison of centrifuged liquid based cytology method with conventional brush cytology in oral lesions. Eur J Gen Dent 2012;1:192-196

20. Greer RO Jr, Poulson TC. Oral tissue alterations associated with the use of smokeless tobacco by teenagers. Part I. Clinical findings. Oral Surg Oral Med Oral Pathol 1983;56:275-284.

21. Aruna DS, Prasad KVV, Shavi GR, Ariga J, Rajesh G, Krishna M. Retrospective study on risk habits among oral cancer patients in Karnataka Cancer Therapy and Research Institute, Hubli, India. Asian Pacific J Cancer Prev 2011;12: 1561-1566.

22. Gupta B, Johnson NW. Systematic review and meta-analysis of association of smokeless tobacco and of betel quid without tobacco with incidence of oral cancer in South Asia and the Pacific. PLoS ONE 2014;9:e113385. 